\title{
Gestação com o Auxílio das Técnicas de Reprodução Assistida: Uma Revisão da Literatura
}

\author{
Lia Mara Netto Dornelles", Isabela Machado da Silva, \& Rita de Cássia Sobreira Lopes \\ Universidade Federal do Rio Grande do Sul, Porto Alegre, Brasil
}

\begin{abstract}
RESUMO
Neste artigo, são revisadas e discutidas pesquisas que investigaram a gestação em casais que conceberam com o auxílio de técnicas de reprodução assistida (TRA). Os resultados obtidos têm sugerido semelhanças entre a resposta emocional desses casais e daqueles que conceberam naturalmente, sendo que o sucesso no tratamento contribuiria para uma melhora nos índices de depressão e ansiedade. Não têm sido identificadas diferenças no que se refere ao apego ao bebê. No entanto, a preocupação de que algo possa acontecer à gravidez ou ao bebê destaca-se entre os casais TRA. Defende-se a necessidade de pesquisas que investiguem a experiência do casal como um todo, bem como a utilização de métodos alternativos de coleta e análise dos dados, como entrevistas abertas e análises qualitativas.
\end{abstract}

Palavras-chave: reprodução assistida; gestação; maternidade; paternidade.

\section{ABSTRACT \\ Pregnancy Through Assisted Reproduction Techniques: A Literature Review}

This paper reviews and discusses studies that investigated pregnancy in couples who conceived through assisted reproduction techniques (ART). The results suggest similarities between the emotional response of ART couples and the ones who conceived spontaneously. Improvements are observed in cases of depression and anxiety when the treatment succeeds. Differences in the attachment to the baby were not identified. However, the fear of what might happen to pregnancy or to the baby is outstanding among ART couples. The need for researches that investigate the couples' experience as a whole is emphasized, as well as the use of alternative ways of collecting and analyzing data, such as open-ended interviews and qualitative analysis.

Keywords: assisted reproduction; pregnancy; motherhood; fatherhood.

O processo reprodutivo possui implicações psicológicas, sociais e culturais, que conferem à reprodução um significado central na vida das pessoas. Portanto, quando o projeto parental não pode se realizar devido a questões de infertilidade em um dos cônjuges ou em ambos, exige-se um amplo processo de reorganização, tanto por parte do indivíduo como do casal (Balmaceda \& cols., 2001). Considerada um evento estressor, a infertilidade tende a promover um impacto significativo no mundo psíquico dos indivíduos inférteis (Balmaceda \& cols., 2001; Makuch, 2001; Ribeiro, 2004).
No entanto, o panorama da reprodução humana frente ao diagnóstico de infertilidade mudou drasticamente a partir do século XX. Se o projeto parental é inviabilizado, casais inférteis podem recorrer às Técnicas de Reprodução Assistida (TRA) em busca da realização do desejo de maternidade e paternidade. Dentre essas técnicas, encontram-se a Fertilização in vitro (FIV), a Inseminação Intrauterina, a Injeção Intracitoplasmática de Espermatozóide (ICSI), a Doação de Gametas e a Gestação Substitutiva (Balmaceda \& cols., 2001; Ferriani \& Navarro, 2004; Passos, Almeida,

\footnotetext{
Endereço para correspondência: Lia Mara Netto Dornelles, Instituto de Psicologia, Universidade Federal do Rio Grande do Sul, Rua Ramiro Barcelos, 2600, sala 108, Porto Alegre, RS, CEP 90035-003. E-mail: liamaradornelles@ gmail.com.

As autoras agradecem o apoio do CNPq, da FAPERGS e da CAPES.
} 
\& Fagundes, 2007). Como tratamento, essas técnicas tendem a ocasionar um desgaste físico e psíquico e a despertar vivências de angústia, esperança, tensão e frustração (Daniluk, 2001).

Embora as características biológicas de uma gravidez decorrente da utilização das TRA sejam semelhantes aos de gestações decorrentes de concepcão natural, deve-se considerar que, quando uma gestação ocorre após uma prolongada experiência de infertilidade, o angustiante tempo de espera pela gravidez mobiliza sentimentos que podem afetar a forma como será exercida a maternidade/paternidade (Ribeiro, 2004). Nesses casos, a gestação constitui uma etapa de transição entre a experiência de infertilidade e a experiência da maternidade/paternidade potencial (Balmaceda \& cols., 2001). Apesar de a gestação representar a realização de um sonho, trata-se de período no qual novas vivências e desafios se impõem ao casal.

Dessa forma, este artigo tem como objetivo revisar e discutir pesquisas que investigaram o período da gravidez em casais que conceberam com o auxílio das TRA. Será examinado o método desses estudos, assim como o que se constatou a respeito de diferentes aspectos dessa experiência, tais como a resposta emocional dos casais e suas atitudes e expectativas em relação à gestação.

\section{MÉTODO}

Realizaram-se buscas em bases de dados, como o PsycInfo e o Ovid-Medline, com o objetivo de acessar artigos que investigaram a gestação em casais que conceberam com o auxílio das TRA. Os termos reproductive technology, assisted reproduction, in-vitro fertilization, artificial insemination e ICSI foram combinados a pregnancy, parent, family, child e successful. Realizaram-se, ainda, buscas por artigos nacionais no Index-Psi, no Scielo e no Pepsic, utilizandose os termos "tecnologias reprodutivas" e "reprodução assistida". A partir dessa busca, acessaram-se 31 artigos, na íntegra, que se adequaram aos propósitos deste estudo, todos internacionais.

\section{RESULTADOS}

A seguir, os resultados obtidos a partir dessa busca serão discutidos quanto (a) ao método adotado por esses estudos, (b) à resposta emocional dos futuros pais frente à gravidez e (c) às suas atitudes e expectativas em relação à gravidez e ao bebê. Essas categorias foram selecionadas tendo em vista os temas predominantemente abordados pelos estudos nessa área e o propósito deste trabalho de fornecer subsídios para uma discussão a respeito da experiência da gravidez nesse contexto.

\section{a) Método adotado pelos estudos que investigaram o tema}

Ao revisar os 31 artigos encontrados, constatou-se que diversos deles são derivados de projetos maiores desenvolvidos por determinados grupos de pesquisa. Os achados desses projetos foram publicados em diferentes artigos, constituindo análises parciais dos dados obtidos a partir da aplicação de um mesmo método. Considerando que esta seção tem como objetivo uma análise metodológica, optou-se por agrupar aqui esses artigos que fazem parte de um mesmo projeto como forma de evitar a repetição de informações e o enviesamento das análises, procedimento este já adotado em outros estudos de revisão sobre o tema (Hammarberg, Fisher, \& Winter, 2008). Atribuiu-se a cada grupo uma mesma nomenclatura, conforme pode ser observado na Tabela 1. Somando-se esses grupos aos demais artigos encontrados, totalizaram-se 18 estudos cujo método será analisado a seguir.

Identificou-se uma tendência a investigar a gravidez de casais que conceberam com o auxílio das TRA a partir de sua comparação com casais que conceberam naturalmente. Essa estratégia esteve presente em 12 estudos. No entanto, foram identificadas também comparações entre outros grupos, conforme pode ser observado na Tabela 2. Apenas um dos estudos não trouxe qualquer tipo de comparação: um estudo qualitativo desenvolvido por Hershberger (2007), que investigou a experiência de gestantes que conceberam com o auxílio da doação de gametas. 
Tabela 1

Grupos de Artigos Derivados de Projetos Comuns

\begin{tabular}{|c|c|}
\hline Artigos & Nomenclatura do grupo \\
\hline Monti e cols. (2008), Monti e cols. (2009); Agostini e cols. (2009) & Monti e cols. (2008-2009) \\
\hline $\begin{array}{l}\text { McMahon, Ungerer, Beaurepaire, Tennant e Saunders (1997); } \\
\text { McMahon, Tennant, Ungerer e Saunders (1999); } \\
\text { Cohen, McMahon, Tennant, Saunders e Leslie (2000) }\end{array}$ & McMahon e cols. (1997-2000) \\
\hline $\begin{array}{l}\text { Hjelmstedt, Widström, Wramsby, Matthiesen e Collins (2003); } \\
\text { Hjelmstedt, Widström, Wramsby e Collins (2003); } \\
\text { Hjelmstedt, Widtröm, Wramsby e Collins (2004); } \\
\text { Hjelmstedt, Widström e Collins (2006); } \\
\text { Hjelmstedt, Widström e Collins (2007) }\end{array}$ & Hjelmstedt e cols. (2003-2008) \\
\hline $\begin{array}{l}\text { Repokari e cols. (2005); Poikkeus e cols. (2006); } \\
\text { Vilska e cols. (2009) }\end{array}$ & Repokari e cols. (2005-2009) \\
\hline $\begin{array}{l}\text { Glazebrook, Cox, Oates e Ndukwe (1999); } \\
\text { Cox, Glazebrook, Sheard, Ndukwe e Oates (2006) }\end{array}$ & Glazebrook e cols. (1999-2006) \\
\hline $\begin{array}{l}\text { Lepecka-Klusek e Jakiel (2007); } \\
\text { Lepecka-Klusek e Jakiel (2009) }\end{array}$ & Lepecka-Klusek e Jakiel (2007-2009) \\
\hline
\end{tabular}

Tabela 2

Comparativos Presentes nos Estudos

\begin{tabular}{|c|c|c|}
\hline Comparativos & Estudos & $\%(n)$ \\
\hline $\begin{array}{l}\text { Casais que conceberam com o auxílio } \\
\text { das TRA } \\
\text { Casais que conceberam naturalmente }\end{array}$ & $\begin{array}{l}\text { Balen, Naaktgeboren e Trimbos-Kemper (1996); Fischer, Hammarberg, } \\
\text { Gordon e Baker (2008); Glazebrook e cols. (1999-2006); Harf-Kashdaei } \\
\text { e Kaitz (2007); Hjelmstedt e cols. (2003-2008); Kloch e Greenfeld (2000); } \\
\text { Lepecka-Klusek e Jakiel (2007-2009); McMahon e cols. (1997-2000); } \\
\text { Monti e cols. (2008-2009); Repokari e cols. (2005-2009); Stanton e } \\
\text { Golombok (1993); Ulrich, Gagel, Hemmerling, Pastor e Kentenich (2004) }\end{array}$ & $\begin{array}{c}66,6 \% \\
(12)\end{array}$ \\
\hline $\begin{array}{l}\text { Casais que obtiveram sucesso no trata- } \\
\text { mento } \\
\text { Casais que não engravidaram }\end{array}$ & $\begin{array}{l}\text { Holter, Anderheim, Bergh e Möller (2006); Slade, Emery e Lieberman } \\
\text { (1997); Verhaak e cols. (2001); Verhaak, Smeenk, Minnen, Kremer e } \\
\text { Kraaimaat (2005) }\end{array}$ & $\begin{array}{c}22,22 \% \\
\quad(4)\end{array}$ \\
\hline $\begin{array}{l}\text { Casais que esperavam gêmeos } \\
\qquad \mathrm{X} \\
\text { Casais que esperavam apenas um bebê }\end{array}$ & Glazebrook e cols. (1999-2006); Repokari e cols. (2005-2009) & $\begin{array}{c}11,11 \% \\
(2)\end{array}$ \\
\hline Casais que recorreram a diferentes TRA & Balen e cols. (1996); Lepecka-Klusek e Jakiel (2007-2009) & $\begin{array}{c}11,11 \% \\
(2)\end{array}$ \\
\hline Casais mais velhos $X$ Casais mais jovens & McMahon, Gibson, Allen e Saunders (2007) & $\begin{array}{c}5,55 \% \\
(1)\end{array}$ \\
\hline
\end{tabular}


Apenas dois dos estudos revisados são retrospectivos (Balen \& cols., 1996; Lepecka-Kluseck \& Jakiel, 2007-2009). Nos demais, os dados foram coletados durante a própria gestação. Conforme destacam Lepecka-Klusek \& Jakiel (2009), os dados obtidos por essas duas abordagens são potencialmente distintos, pois a experiência do parto e o contato com o recémnascido tendem a influenciar a forma como a mãe se refere ao período da gestação.

Entre os estudos prospectivos, os dados foram coletados em diferentes momentos da gestação. O terceiro trimestre de gravidez foi o período que recebeu maior atenção dos pesquisadores, sendo que 12 estudos coletaram ao menos parte de seus dados nesse momento (Fischer \& cols., 2008; Glazebrook \& cols., 1999-2006; Harf-Kashdaei \& Kaitz, 2007; Hjelmstedt \& cols., 2003-2007; Kloch \& Greenfeld, 2000; McMahon \& cols., 1997-2000; Mc Mahon \& cols., 2007; Monti \& cols., 2008-2009; Slade \& cols., 1997; Stanton \& Golombok, 1993; Ulrich \& cols., 2004; Verhaak \& cols., 2005). As mudanças emocionais ocorridas no decorrer da gestação foram o foco de alguns estudos, que coletaram seus dados em diferentes momentos (Fischer \& cols., 2008; Glazebrook \& cols., 1999/2006; Hjelmestedt \& cols., 2003-2008; Kloch \& Greenfeld, 2000; McMahon \& cols., 2007). Destaca-se a importância de atentar ao período da gravidez em que os casais se encontram, uma vez que, como bem pontuam Brazelton e Cramer (1992), ao longo da gestação, são vivenciados diferentes estágios, cada um marcado por características específicas que refletem o momento em que se encontra a gestante. Dessa forma, a experiência de uma mulher que se encontra no primeiro trimestre de gestação, quando ainda está assimilando a ideia da gravidez, tende a ser diferente daquela que já está no segundo ou terceiro trimestre, o que pode influenciar os resultados obtidos. Nesse contexto, estudos longitudinais mostram-se bastante úteis para clarificar essas mudanças.

No que se refere aos instrumentos utilizados, a grande maioria dos estudos recorreu a escalas padronizadas. Em apenas seis foram realizadas entrevistas abertas com os participantes, as quais enfocaram as representações parentais acerca de si mesmo, do cônjuge e do bebê (Harf-Kashdaei \& Kaitz, 2007; Monti \& cols., 2008-2009), o ajustamento à gravidez, as atitudes em relação ao bebê, as expectativas em relação ao nascimento e à maternidade/paternidade (McMahon \& cols., 1997-2000), a história do casal, seu desejo em relação à vinda do filho, as repercussões emocionais da infertilidade (Ulrich \& cols., 2004) e a experiência da gravidez e do tratamento (Hershberger, 2007; Hjelmstedt \& cols., 2003-2007). Cohen e cols. (2000) destacaram a importância da realização de entrevistas abertas ao afirmarem que as mesmas lhes possibilitaram reconhecer especificidades presentes no contexto da reprodução assistida, não identificadas pelos demais instrumentos utilizados. No entanto, somente Hershberger (2007) adotou a entrevista como único instrumento de coleta de dados. A distribuição dos artigos segundo os instrumentos utilizados pode ser constatada na Figura 1.

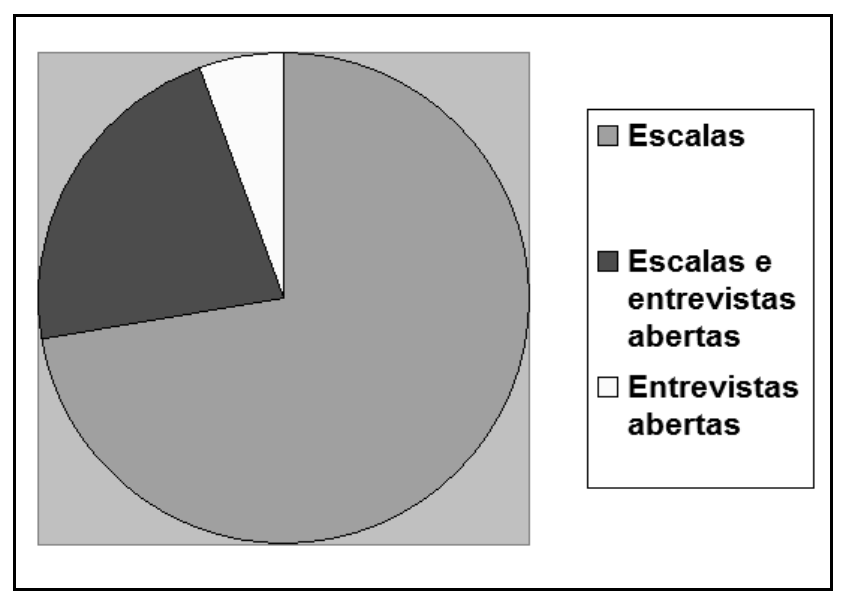

Figura 1. Instrumentos utilizados. 
De forma coerente ao que se constatou na análise dos instrumentos utilizados, em 15 dos estudos, os dados foram submetidos a análises quantitativas. Estas estiveram presentes mesmo na análise dos dados oriundos de entrevistas abertas com os participantes, as quais foram codificadas para análise. Dois estudos associaram análises qualitativas e quantitativas como forma de ampliar a compreensão dos dados e de discuti-los (Hjelmstedt \& cols., 2003-2007; McMahon \& cols., 1997-2000). O único estudo caracterizado por uma análise puramente qualitativa foi o de Hershberger (2007), que adotou um referencial fenomenológico. A distribuição segundo o método pode ser observada na Figura 2.

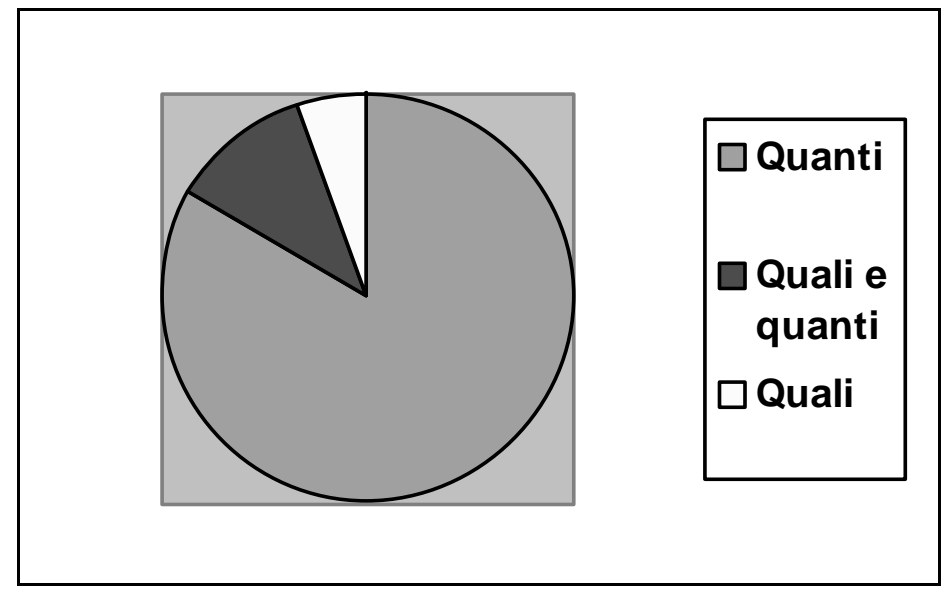

Figura 2. Análise dos dados.

A composição da amostra é outro fator que merece ser discutido. Embora já se identifique uma tendência quanto à realização de estudos que contem com a participação tanto das gestantes como de seus companheiros, em oito, apenas as mulheres participaram (Fisher \& cols., 2008; Harf-Kashdaei \& Kaitz, 2007; Hershberger, 2007; Kloch \& Greenfeld, 2000; Lepecka-Klusek \& Jakiel, 2007-2009; Stanton \& Golombok, 1993; Verhaak \& cols., 2001). A importância de atentar para as vivências masculinas é demonstrada por autoras como Seger-Jacobs (2006) e Weiss (2006), que afirmam que, embora os homens respondam de forma diferente à infertilidade, este é um evento que também os afeta profundamente. Apesar de se considerar que os homens possam ser mais resistentes a participar de estudos como esses, em função da exposição envolvida e do estigma que a infertilidade ainda traz consigo, destaca-se a importância de sua participação para a elaboração de estratégias de apoio a essa população.

\section{b) Respostas Emocionais}

Estudos que compararam casais que obtiveram sucesso e aqueles que não conceberam após as TRA investigaram diferentes aspectos de suas respostas emocionais. Holter e cols. (2006) observaram, duas semanas após o teste de gravidez ou a ocorrência da menstruação, que as mulheres que conseguiram engravidar apresentaram menores índices de raiva, frustração, sensação de isolamento, culpa e depressão. Por outro lado, relataram maior sentimento de sucesso, alegria e satisfação. Em seu estudo com mulheres que se submetiam à primeira experiência com as TRA, Verhaak e cols. (2001) não constataram, um mês após o tratamento, diferença significativa entre ambos os grupos no que se refere aos níveis de ansiedade e depressão. Os autores levantaram a hipótese de que esse achado possa se dever ao fato de as mulheres mais ansiosas não terem se disposto a participar ou terem abandonado o estudo. No entanto, em estudo com casais que se submeteram a sucessivas tentativas (Verhaak \& cols., 2005), diferenças foram identificadas: as mulheres que não obtiveram sucesso no tratamento apresentaram uma elevação nos níveis de ansiedade e depressão um mês após o tratamento, ao contrário daquelas que engravidaram, as quais experimentaram uma redução desses índices. Esses achados mantiveram-se seis meses após o término do tratamento, assemelhando-se ao que foi constatado por Slade e cols. (1997). Observa-se, portanto, uma tendência, entre os estudos, de apontarem para uma redução dos níveis de ansiedade e depressão após o sucesso do tratamento. 
Também foram comparados diferentes aspectos das respostas emocionais de casais que conceberam com o auxílio das TRA e de casais que conceberam naturalmente. A maior parte dos estudos não constatou diferenças entre esses grupos, no que se refere à ansiedade global (Cohen \& cols., 2000; Cox \& cols., 2006; Harf-Kashdaei \& Kaitz, 2007; Klock \& Greenfeld, 2000; McMahon \& cols., 1997, 2007; Poikkeus \& cols., 2006; Stanton \& Golombok, 1993). Para Cohen e cols. (2000) e Stanton e Golombok (1993), o nível de ansiedade desses grupos apresentou-se dentro da normalidade, sendo que se identificou um decréscimo ao longo da gestação (Cox \& cols., 2006; Stanton \& Golombok, 1993).

Monti e cols. (2008), no entanto, constataram níveis mais elevados de ansiedade latente entre as mulheres que conceberam com o auxílio das TRA. Esse tipo de ansiedade refere-se a uma medida da ansiedade inconsciente e não manifesta, e foi avaliada a partir de uma escala que tem como objetivo mensurar diferencialmente tanto os níveis desse tipo de ansiedade como os níveis de ansiedade manifesta, ou seja, sintomática e consciente. Segundo os autores, esse achado poderia estar relacionado ao caráter especial das gestações decorrentes de TRA. Slade e cols. (1997) ressaltam, no entanto, a importância de, ao investigar a ansiedade desses casais, compreender seu conteúdo e foco.

No que se refere aos níveis de depressão, também não foi encontrada diferença significativa, entre os grupos, para a maior parte dos estudos (Glazebrook \& cols., 2000; Klock \& Greenfeld, 2000; Harf-Kashdaei \& Kaitz, 2007). O estudo de Repokari e cols. (2005) observou, inclusive, que homens e mulheres do grupo TRA apresentaram menor incidência de sintomas de depressão. Klock e Greenfeld (2000) atribuíram a semelhança entre os grupos a uma possível interferência causada pela forma de recrutamento das participantes. Segundo os autores, somente indivíduos menos ansiosos se ofereceriam para participar de estudos como esse, sendo que os que participam talvez pouco expressem sentimentos que consideram negativos.

Uma exceção é o estudo de Monti e cols. (2009), em que se encontraram escores mais elevados de depressão entre as mulheres TRA. Segundo esses autores, é possível que, embora tenham engravidado, essas mulheres permaneçam afetadas pela sua inabilidade de procriar espontaneamente, visto que a maternidade envolve tanto questões biológicas como psicológicas. Deve-se considerar, no entanto, que o número de potenciais participantes que se recusou a participar deste estudo foi muito superior ao dos demais, o que pode ter levado a um enviezamento da amostra.

Outras dimensões do humor foram também avaliadas, tais como vigor, fadiga, hostilidade e confusão (Fischer \& cols., 2008). As mulheres que conceberam com o auxílio das TRA apresentaram menores índices nas escalas que avaliaram hostilidade, fadiga e transtornos do humor em geral, quando comparadas a normas populacionais de gestantes, demonstrando, ainda, maior vigor. Os autores atribuem parte desses achados ao fato de essas mulheres serem mais velhas, já possuírem moradia própria e emprego estável, além da gestação ter sido muito desejada. Acrescentam, porém, que esses resultados indicam que a maternidade pode ser vista por essas mulheres de forma idealizada.

Em relação à autoestima, alguns estudos apontaram semelhanças entre os grupos (Cox \& cols., 2006; Klock \& Greenfeld, 2000), sugerindo, inclusive, um aumento da autoestima no grupo TRA ao longo da gestação. Segundo Klock e Greenfeld (2000), esses resultados podem estar relacionados à satisfação por ter engravidado e ao fato de que a infertilidade foi em sua maioria atribuída aos homens. Cohen e cols. (2000), no entanto, encontraram menor autoestima entre os homens do grupo FIV na trigésima semana de gestação. De acordo com os autores, é possível que a proximidade do nascimento traga maiores preocupações e incertezas a esses pais.

Uma importante questão a ser considerada no contexto das TRA refere-se à ocorrência de gestações múltiplas. De acordo com Glazebrook e cols. (2000), nos casos em que há gestação múltipla decorrente de TRA, o nível de ansiedade tende a ser superior ao constatado em gestações únicas, também decorrentes de TRA, ou em gestações naturais. Vilska e cols. (2009), por sua vez, encontraram maiores índices de depressão, ansiedade e de dificuldades de sono em casais que esperavam gêmeos do que naqueles que esperavam apenas um bebê, independentemente de sua forma de concepção. Assim, esses autores concluíram que a saúde mental durante esse momento não sofre impacto negativo das TRA, mas da maternidade/paternidade de gêmeos. 


\section{c) Atitudes e expectativas em relação à gravidez $\mathrm{e}$ ao bebê}

Embora autores como Stanton e Golombok (1993) e Ulrich e cols. (2004) não tenham encontrado diferenças entre casais que conceberam naturalmente $\mathrm{e}$ casais que engravidaram com o auxílio das TRA no que se refere à vivência da gestação, outros estudos sugeriram algumas especificidades que podem estar presentes em uma gravidez deste tipo. McMahon e cols. (1999), Kloch e Greenfeld (2000) e Hjelmstedt, Widström, Wramsby e Collins (2003) encontraram evidências de que a gravidez tende a ser descrita pelas mulheres do grupo TRA como uma experiência mais gratificante e satisfatória. Essas mulheres relataram considerar o desconforto advindo da gravidez como mais válido, demonstrando maior aceitação em relação às mudanças corporais vivenciadas na gestação (Hjelmstedt, Widström, Wramsby, \& Collins, 2003). Participantes do estudo de McMahon e cols. (1999) referiram que as dificuldades da gestação seriam secundárias frente à alegria de conceber e que a experiência da infertilidade teria enriquecido a vivência da gestação. No entanto, embora esses autores concebam que esses dados possam ser fruto de um maior preparo emocional para lidar com essas dificuldades em função da experiência do tratamento, eles também levantam a possibilidade de uma possível idealização da gestação e da consequente negação das dificuldades (Hjelmstedt, Widström, Wramsby, \& Collins, 2003; McMahon \& cols., 1999).

Estudos retrospectivos, como os desenvolvidos por Lepecka-Klusek e Jakiel (2007, 2009) e Balen e cols. (1996) chegaram a resultados que apontam em uma direção oposta. De acordo com esses autores, as mulheres que conceberam com o auxílio das TRA tenderam a identificar maiores dificuldades em suas gestações, vivenciando-as como mais estressantes. Segundo Lepecka-Klusek e Jakiel (2007), toda a espera pela gravidez e a falta de confiança nas técnicas adotadas podem contribuir para um estado de ansiedade e receio em relação ao andamento da gravidez. Além disso, o fato de essas mulheres considerarem sua gestação como muito valiosa e única tenderia a acrescentar um estresse adicional ao processo (Balen \& cols., 1996). Deve-se considerar, no entanto, a natureza retrospectiva desses estudos ao analisar essa aparente divergência de resultados. Se, de fato, a gestação é vivenciada de forma mais idealizada por essas mulheres, é possível que elas se frustrem ao se depararem com a realidade da maternidade, o que poderia leválas a recordar o período da gestação como mais estressante, ao contrário do que tenderia a ser relatado por gestantes que estão vivenciando a gravidez justamente no momento em que participam do estudo.

No que se refere à vivência dos futuros pais, Hjelmstedt, Widström, Wramsby, Matthiesen e Collins (2003) constataram que os homens do grupo TRA apresentaram menor ambivalência em relação à gestação do que o grupo controle no início da gravidez. No estudo de Balen e cols. (1996), por sua vez, esses homens referiram-se à gravidez de suas companheiras como mais agradáveis e excepcionais. Segundo esses autores, a experiência da infertilidade contribuiria para um envolvimento mais forte dos futuros pais com a gravidez. Agostini e cols. (2009) constataram, no entanto, que os homens de seu estudo mostraram-se menos envolvidos com a gravidez do que suas futuras esposas. De acordo com esses autores, as mulheres tendem a se envolver mais em função de todo o processo ocorrer em seus corpos.

Uma análise mais cuidadosa dos achados obtidos, porém, demonstra a necessidade de considerar a complexidade dessa experiência, não a classificando apenas como mais satisfatória e agradável, por um lado, ou como mais estressante e difícil, por outro. Os dados qualitativos obtidos por Hjelmstedt, Widström, Wramsby e Collins, (2003), por exemplo, trouxeram tanto relatos de uma experiência denominada mais positiva da gravidez, de maior preparo para sua vivência e de maior envolvimento por parte dos pais como de um sentimento de que essa gravidez representa a única possibilidade de ter um filho, de maior foco e controle da gestação e de não se permitir comemorar a gravidez. Hjelmstedt, Widström, Wramsby, Matthiesen e Collins (2003) destacaram, ainda, que, embora os homens do grupo TRA tenham se mostrado menos ambivalentes, existem, em ambos os grupos, aqueles que apresentaram índices elevados de ambivalência. Dessa forma, ressaltaram que os profissionais da área da saúde não devem ter a expectativa de que casais que engravidam com o auxílio das TRA expressem apenas sentimentos considerados positivos em relação à gestação, especialmente tendo em vista que certo nível de ambivalência é característico ao período gestacional.

Em um estudo sobre as representações parentais, Agostini e cols. (2009), identificaram que as mulheres do grupo TRA mostraram-se mais envolvidas e relata- 
ram mais fantasias do que as do grupo controle. Segundo os autores, esse é um dado esperado, em função do tempo durante o qual se aguardou por essa gravidez e de sua consequente valorização. No entanto, esses achados foram relacionados a níveis mais elevados de ansiedade, uma vez que a preocupação de que algo aconteça com esse bebê tão esperado é maior. Além disso, essas mulheres apresentaram representações mais ambivalentes, ao contrário do grupo controle, em que predominaram representações mais integradas. Haveria, dessa forma, uma dificuldade em integrar elementos denominados positivos e negativos, assim como reais e imaginários.

Alguns artigos investigaram, ainda, fatores capazes de influenciar a forma como a gestação é percebida e que devem ser considerados ao se estudar essa questão. Lepecka-Klusek e Jakiel $(2007,2009)$ constataram que mulheres mais jovens, de elevado nível econômico e educacional, que passaram por complicações médicas, que fizeram uso de TRA mais sofisticadas ou que tiveram maiores dificuldades para custear o tratamento tenderiam a perceber a gestação como mais difícil. Segundo McMahon e cols. (1999), o número de ciclos a que o casal se submeteu na tentativa de engravidar seria outro fator relevante, sendo que um número mais elevado estaria relacionado a uma percepção da gravidez como um evento mais gratificante. Em outro estudo, McMahon e cols. (2007) ressaltaram, ainda, a importância da idade: mães mais velhas estariam, segundo esses autores, menos sujeitas a idealizarem a maternidade.

Outra questão importante enfocada pelos estudos revisados refere-se às preocupações vivenciadas durante a gestação. No estudo de Kloch e Greenfeld (2000), foram investigados diversos tipos de preocupações, sendo que as mulheres do grupo TRA relataram que se sentiam menos preocupadas em relação aos seguintes aspectos: mudança de peso, distanciamento do marido, restrição da independência, aumentos nos gastos e perdas de rendimentos em função da gravidez.

Por outro lado, parece haver certo consenso de que essas mães apresentariam índices mais elevados de ansiedades específicas à gestação e ao bem-estar do bebê. Em um estudo longitudinal, Hjelmstedt Widström, Wramsby e Collins (2003) observaram que o medo da perda da gravidez mostrou-se superior entre as mulheres do grupo TRA ao longo de toda a gestação, tendendo, porém, a decrescer com o passar do tempo. Por outro lado, houve, entre essas mulheres, um crescimento da ansiedade em relação à saúde do bebê com o passar do tempo. Os autores sugeriram que essas mulheres só conseguiriam se preocupar com os bebês após se sentirem seguras quanto à continuidade da gravidez. McMahon e cols. (1997) também defenderam que ansiedades específicas persistiriam entre essas mulheres no decorrer de toda a gravidez. Esses autores constataram que, no terceiro trimestre de gravidez, as mulheres do grupo TRA mostraram-se mais ansiosas em relação à saúde e a possíveis deficiências do filho. Além disso, elas também demoraram mais para confiarem na continuidade de sua gravidez, adiando por mais tempo a revelação de que estavam grávidas, e expressaram maior preocupação em relação ao parto e a possíveis separações do bebê após o nascimento. McMahon e cols. (1999) identificaram, ainda, que essas mulheres tenderiam a buscar menos informações sobre a gravidez do que o grupo controle, o que é explicado pelos autores como uma forma de reduzir a ansiedade ao evitar informações a respeito de possíveis complicações.

No que se refere aos futuros pais, Hjelmstedt e cols, (2003) também constataram uma maior ansiedade quanto à perda da gravidez, sendo que, no entanto, a mesma tenderia a decair, durante a gestação, de forma muito mais acentuada do que o observado no grupo controle. Os homens do grupo TRA mostraramse, ainda, mais ansiosos em relação à possibilidade de o bebê se machucar durante o parto.

Uma exceção frente a esses resultados é apresentada no artigo de Poikkeus e cols. (2006). De acordo com esses autores, não foram encontradas diferenças entre os grupos de casais que conceberam naturalmente e daqueles que recorreram às TRA no que se refere à ansiedade em relação à gravidez e ao medo do parto. No entanto, os próprios autores ressaltam que o fato de os dados terem sido coletados no segundo trimestre de gestação pode ter influenciado, uma vez que o nível de bem-estar geral tenderia a ser superior nessa fase.

Investigaram-se também fatores capazes de influenciar a vivência dessas ansiedades específicas em relação à gravidez ou ao bebê. De acordo com Hjelmstedt, Widström, Wramsby e Collins (2003) e Hjelmstedt, Widström, Wramsby, Matthiesen e Collins (2003), o impacto da infertilidade seria um fator a ser considerado, uma vez que indivíduos que apresentaram escores elevados em uma escala que avaliou essa questão tenderam a se mostrarem mais ansiosos. $\mathrm{O}$ 
tempo de coabitação, a tendência geral à ansiedade e o nível social também foram questões que se mostraram relevantes de acordo com os dados obtidos por esses autores. Segundo Poikkeus e cols. (2006), o tempo da relação e o número de tentativas de tratamento a que o casal se submeteu anteriormente estariam inversamente relacionados à ansiedade exibida, ao contrário do tempo de duração da infertilidade. McMahon e cols. (1997) também identificaram a importância do número de tentativas prévias, sendo que as mães com dois, três ou mais ciclos mostraram-se mais preocupadas, ao contrário das com apenas um ciclo, que se assemelharam ao grupo controle. Os autores destacaram a importância de considerar a experiência do tratamento para que não se percam as especificidades da realidade vivenciada por casais que se submeteram a essas técnicas repetidas vezes. No entanto, sugeriram que os menores níveis de ansiedade demonstrados por mães que haviam se submetido a apenas um ciclo poderiam estar relacionados a uma estratégia de negação do estresse, motivada pelo desejo de convencerem aos outros e a si mesmas de sua normalidade. Essa hipótese é reforçada pelos dados de que essas mulheres apresentaram maiores índices de supressão de ansiedade em uma escala sobre controle emocional. A técnica utilizada também se mostrou uma importante questão a ser considerada, uma vez que certas preocupações mostraram-se diretamente relacionadas ao procedimento adotado. Hershberger (2007), por exemplo, constatou que mulheres que conceberam a partir de gametas doados referiram receio quanto a futuros contatos com os doadores, quanto ao desconhecimento da história genética da criança no caso de doadores desconhecidos e quanto a dificuldades no relacionamento familiar no caso em que os doadores são parentes. No que se refere à idade, Hershberger (2007) identificou preocupações em relação aos seus efeitos sobre o exercício da maternidade. No entanto, McMahon e cols. (2007) não observaram diferenças entre mulheres mais jovens e mais velhas que conceberam com o auxílio das TRA no que refere a ansiedades específicas à saúde do bebê.

A relação dos pais com o feto também foi uma questão a receber a atenção dos pesquisadores da área. De forma geral, não se encontraram diferenças nas medidas de apego parental ao feto entre casais que recorreram às TRA e casais que conceberam naturalmente (Cohen \& cols., 2000; Hjelmstedt \& cols., 2006/2007; McMahon \& cols., 1997; Stanton \& Golombok, 1993). Observou-se, ainda, que esse apego tenderia a aumentar ao longo da gestação (Fisher \& cols., 2008; Hjelmstedt \& cols., 2006-2007). Em seu estudo, Fisher e cols. (2008) constataram, inclusive, que mulheres que conceberam com o auxílio dessas técnicas tenderam a apresentar, já no início da gravidez, um nível de apego comparável ao observado em normas populacionais de mulheres que se encontravam em um estado mais avançado de gestação. Dessa forma, elas demonstrariam uma ligação mais protetora e intensa em relação a seus bebês. Os autores destacaram, no entanto, que sua amostra caracterizou-se pela presença de diversos fatores protetores: alto nível social e educacional, assim como relacionamento conjugal descrito como sensível, apoiador e afetuoso, com baixos índices de coerção e crítica. Além disso, sugeriram a possibilidade de que a gravidez seja idealizada por essas mulheres e de que elas se sintam menos predispostas a expressarem dúvidas e incertezas após tudo o que vivenciaram. Essas mesmas questões são discutidas por Harf-Kashdaei e Kaitz (2007) que, de forma semelhante, constaram que as mulheres do grupo TRA tenderam a demonstrar uma atitude mais positiva em relação a seus bebês, realizando mais afirmações denominadas positivas do que negativas ao se referirem a eles. No grupo controle, houve uma preponderância de afirmações consideradas negativas. No entanto, esses autores afirmam que também há a possibilidade de que esses resultados sejam fruto do desejo por essa gravidez, das habilidades de coping exercitadas frente aos diversos desapontamentos vivenciados ou de uma seleção ocorrida ao longo do tratamento, em que só prosseguiriam os casais mais bem-ajustados.

Apesar desses resultados, McMahon e cols. (1999) identificaram que mulheres que conceberam a partir das TRA relataram conversar menos com seus bebês e adiar o início dos preparativos para recepcioná-los. Os autores afirmaram, porém, que interpretar tais comportamentos como indicativos de menor apego não seria coerente com os demais resultados obtidos. Os mesmos poderiam representar uma tentativa dessas mães de se protegerem contra possíveis decepções caso algo aconteça à gravidez ou ao bebê.

Entre as questões que parecem estar inversamente relacionadas ao apego ao feto está a ambivalência em relação à gestação e traços de personalidade caracterizados por tendência à ansiedade ou ao distanciamento emocional. Por outro lado, a satisfação conjugal estaria diretamente relacionada, ao menos para as mulheres 
(Hjelmstedt \& cols., 2006-2007). Stanton e Golombok (1993) observaram, ainda, que mulheres que apresentaram índices mais elevados em uma escala que avalia atitudes negativas em relação à gravidez, ao parto e aos cuidados com o bebê, tenderam a se mostrar menos apegadas ao feto.

Entre os estudos revisados, poucos abordaram, de forma explícita, as expectativas em relação ao bebê ou ao futuro. Hjelmstedt, Widström, Wramsby e Collins (2003) constataram que mulheres que conceberam com o auxílio das TRA tenderam a considerar com menor frequência que os filhos representariam uma restrição à sua liberdade. De acordo com os autores, tal achado pode tanto indicar que essas mulheres estariam mais dispostas a abrirem mão de sua liberdade como a presença de expectativas irreais. No entanto, os resultados obtidos por McMahon e cols. (1999) não sugerem essa idealização, uma vez que as mulheres desse grupo tenderam a esperar bebês mais difíceis. Segundo os autores, em função de suas experiências, haveria, nessas mulheres, a crença de que poderiam se deparar com dificuldades a qualquer momento, de forma que tais expectativas podem representar uma tentativa de evitar desapontamentos futuros. Referiram, ainda, que essa postura pode representar a disposição a aceitar um filho que não seja perfeito, pois, como afirmou uma participante do estudo desses autores: "aqueles que imploram não podem escolher" (p. 354). No que se refere aos futuros pais, Cohen e cols. (2000) não encontraram diferenças entre o grupo controle e o TRA no que se refere às dificuldades esperadas.

\section{CONSIDERAÇÕES FINAIS}

A gestação, no contexto das TRA, tem como pano de fundo a vivência da infertilidade, uma experiência que demanda a reorganização das metas do casal e a superação de uma série de desafios, tanto referentes ao próprio tratamento como ao sofrimento psíquico vivenciado (Balmaceda \& cols., 2001; Verhaak \& cols., 2005). Trata-se, portanto, de uma gestação que se inicia de forma singular, o que levanta questões acerca de suas especificidades.

Os estudos revisados tendem a sugerir semelhanças na resposta emocional de casais que engravidaram com o auxílio das TRA e daqueles que conceberam naturalmente. Uma melhora nos índices de depressão e ansiedade, entre outros, parece ocorrer quando a gravidez é alcançada. No entanto, foram identificadas também certas particularidades. Embora não tenham sido constatadas diferenças no apego dos futuros pais aos seus bebês, destacaram-se as preocupações e ansiedades vivenciadas por esses casais em relação à gravidez e ao bem-estar do bebê.

Além disso, também se constatou a importância de atentar às diferenças existentes entre os casais. Idade, cultura, nível socioeducacional, experiência do tratamento, ocorrência de gestações múltiplas e utilização de técnicas específicas - como as que envolvem doação de gametas ou procedimentos mais elaborados parecem influenciar a forma como a gestação será vivenciada, devendo, portanto, ser consideradas pelos estudos que abordam essa temática, uma vez que podem influenciar os resultados.

Ao discutirem achados favoráveis aos casais que engravidaram com o auxílio das TRA, os autores parecem dividir-se entre duas possibilidades principais. De um lado, pode haver uma idealização da gestação por parte desses casais, uma tendência a negar as dificuldades vivenciadas em função de a gravidez ter sido tão desejada e batalhada. Por outro, é também possível que, devido à desgastante experiência da infertilidade e do tratamento, esses casais mostrem-se mais preparados para enfrentarem as dificuldades encontradas na gestação. Para a melhor compreensão desse processo, são necessários estudos que investiguem essa experiência em profundidade.

No entanto, é necessário considerar que a maior parte dos estudos baseou-se em escalas padronizadas, que, embora auxiliem a identificação de tendências gerais, dificultam o acesso às especificidades dessa experiência (Cohen \& cols., 2000) e às particularidades de cada casal e sua história. Os estudos encontrados priorizam temas pontuais e isolados, sugerindo a presença ou ausência de sintomas e propondo quantificações e comparações que, isoladamente, são incapazes de abarcar essa experiência.

Outra questão a ser destacada refere-se à tendência de caracterizar certos sentimentos e atitudes como positivos ou negativos sem considerar o contexto em que os mesmos se apresentam. Conforme bem pontuado por Hjelmstedt, Widström, Wramsby, Matthiesen e Collins (2003), a ambivalência faz parte do processo gestacional. Dessa forma, diferentes sentimentos são importantes para a elaboração necessária a esse período, dependendo das experiências específicas vivenciadas por cada casal. É necessário ter em mente que a simples rotulação de sentimentos e atitudes como positivos ou negativos tende a acarretar repercussões 
prejudiciais aos casais, que podem se recriminar injustamente por certos sentimentos, e à própria prática clínica se não houver uma problematização e uma contextualização adequadas. Portanto, é importante que os profissionais que atendem a essa população estejam atentos ao fato de que a experiência da gravidez nesse contexto não é marcada apenas por sentimentos considerados "positivos" (Hjelmstedt, Widström, Wramsby, Matthiesen e Collins, 2003). Somente assim poderão criar espaço para que esses casais também possam trabalhar suas ambivalências e dificuldades, o que tende a ser especialmente difícil considerando que os mesmos podem sentir que não têm o direito de se queixarem após todo o esforço despendido para alcançarem a gravidez (Golombok \& cols., 1996; Hjelmstedt \& cols., 2004; McMahon \& cols., 2003; Ulrich \& cols. 2004).

O complexo processo de tornar-se pai e mãe nesse contexto estende-se para além do que é mensurável. Presença versus ausência, maior intensidade versus menor intensidade, são binômios que não compreendem a totalidade da experiência psíquica vivida pelos casais ao longo desse processo, que se inicia com dor psíquica e coloca o sujeito frente a frente com a sua história de vida. A concepção não é meramente biológica, mas também psíquica (Raphael-Leff, 1997). É preciso compreender com profundidade como se desenrola o processo de tornar-se pai e mãe nesses casais, pois as interações que se estabelecerão na tríade pai-mãe-bebê são essenciais tanto para o desenvolvimento emocional do bebê, bem como para o desenvolvimento dos próprios pais (Colarusso, 1990). Propõe-se, assim, a necessidade de ampliar o uso de técnicas alternativas de análise e coleta de dados - tais como entrevistas abertas e análises qualitativas - para ampliar a compreensão desse tema.

\section{REFERÊNCIAS}

Obs.: Referências precedidas por asterisco indicam estudos incluídos na revisão.

*Agostini, F., Monti, F., Fagandini, P., Liewellyn, L., De Pascallis, D., La Salla, G. B., \& Blickstein, I. (2009). Parental mental representations during late pregnancy and early parenthood following assisted reproductive technology. Journal of Perinatal Medicine, 37, 320-327.

*Balen, F., Naaktgeboren, N., \& Trimbos Kemper, T. C. M. (1996). In-vitro fertilization: The experience of treatment, pregnancy and delivery. Human Reproduction, 11, 95-98.

Balmaceda, R., Fernández, O., Fernández, E., Fabres, V., Huidobro, A., Sepúlveda, J., \& Zegers, F. (2001). Tener un hijo: Conociendo la infertilidad y los caminos para resolverla. Santiago: Publicações Técnicas Mediterrâneo.
Brazelton, T. B. \& Cramer, B. G. (1992). As primeiras relações (M. B. Cipolla Trans.). São Paulo: Martins Fontes.

*Cohen, J., McMahon, C., Tennant, C., Saunders, D., \& Leslie, G., (2000). Psychosocial outcomes for fathers after IVF conception: A controlled prospective investigation from pregnancy to four months post-partum. Reproductive Technologies, 10, 126-130.

Colarusso, C. A. (1990). The third Individuation: The effect of biological parenthood on separation-individuation processes in adulthood. Psychoanalytic Study of the Child, 45, 179-194.

*Cox, S. J., Glazebrook, C., Sheard, C., Ndukwe, G., \& Oates, M. (2006). Maternal self-esteem after successful treatment for infertility. Fertility and Sterility, 85, 84-89.

Daniluk, J. C. (2001). "If we had it to do over again...": Couples' reflections on their experiences of infertility treatment. The Family Journal, 9(2), 122-133.

Ferriani, R. \& Navarro, P. (2004). Protocolos de conduta em infertilidade conjugal. São Paulo: Conexão Brasileira de Computação Gráfica.

*Fisher, J. R. W., Hammarberg, K., \& Baker, G. H. W. (2008). Antenatal mood and fetal attachment after assisted conception. Fertility and Sterility, 89, 1103-1112.

*Glazebrook, C., Cox, S., Oates, M., \& Ndukwe, G. (2000). Psychological adjustment during pregnancy and the postpartum period in single and multiple in vitro fertilization births: A review and preliminary findings from an ongoing study. Reproductive Technologies, 10, 112-119.

Golombok, S., Brewaeys, A., Cook, R., Giavazzi, M. T., Guerra, D., Mantovani, A., Hall, E. V., Crosignani, P. G., \& Dexeus, S. (1996). The European study of assisted reproduction families: Family functioning and child development. Human Reproduction, 11, 2324-2331.

Hammarberg, K., Fisher, J. R. W., \& Wynter, K. H. (2008). Psychological and social aspects of pregnancy and early parenting after assisted conception: A systematic review. Human Reproduction Update, 14 , 395-414.

*Harf-Kashdaei, E., \& Kaitz, M. (2007). Antenatal moods regarding self, baby, and spouse among women who conceived by in vitro fertilization. Fertility and Sterility, 87, 1306-1313.

*Hershherger, P. E. (2007). Pregnant donor oocyte recipient women describe their lived experience of establishing the "family lexicon". Journal of Obstetric, Gynecologic \& Neonatal Nursing, 36, 161-167.

*Hjelmstedt, A, Widström, A. M., Wramsby, H., \& Collins, A. (2004). Emotional adaptation following successful in vitro fertilization. Fertility and Sterility, 81, 1254-1264.

*Hjelmstedt, A., Widström, A. M., \& Collins, A. (2006). Psychological correlates of prenatal attachment in women who conceived after in vitro fertilization and women who conceived naturally. Birth, 33, 303-310.

*Hjelmstedt, A., Widström, A. M., \& Collins, A. (2007). Prenatal attachment in swedish IVF fathers and controls. Journal of Reproductive and Infant Psychology, 25, 296-307.

*Hjelmstedt, A., Widström, A. M., Wramsby, H., \& Collins, A. (2003). Patterns of emotional responses to pregnancy and attitudes to parenthood among IVF couples: A longitudinal study. Journal of Psychosomatic Obstetrics and Gynecology, 24, 153162.

*Hjelmstedt, A., Widströn, A., Wramsby, H., Matthiesen, A., \& Collins, A. (2003). Personality factors and emotional response to pregnancy among IVF couples in early pregnancy: A com- 
parative study. Acta Obstetricia et Gynecologica Scandinavica, $82,152-161$.

*Holter, H., Anderheim, L., Bergh, C., \& Möller, A. (2006). First IVF treatment-short term impact on psychological well-being and the marital relationship. Human Reproduction, 21, 32953302 .

*Klock, S. C., \& Greenfeld, D. A. (2000). Psychological status of in vitro fertilization patients during pregnancy: A longitudinal study. Fertility and Sterility, 73, 1159-1164.

*Lepecka-Klusek, C., \& Jakiel, G. (2007). Difficulties in adaptation to pregnancy following natural conception or use of assisted reproduction techniques: A comparative study. The European Journal of Contraception and Reproductive Health Care, 12, 51-57.

*Lepecka-Klusek, C., \& Jakiel, G. (2009). The socio-economic conditioning of difficulties in adaptation to pregnancy following assisted reproductive techniques. The European Journal of Obstetrics \& Gynecology and Reproductive Biology, 143, 50-54.

Makuch, M. Y. (2001). Vivências de mulheres e homens do programa de fertilização in vitro da UNICAMP. Dissertação de mestrado não-publicada, Universidade Estadual de Campinas.

*McMahon, C. A., Tennant, C., Ungerer, J., \& Saunsders, D. (1999). 'Don't count your chickens': A comparative study of the experience of pregnancy after IVF conception. Journal of Reproductive and Infant Psychology, 17, 345-356.

*McMahon, C. A., Gibson, F. L., Allen, L., \& Saunders, D. (2007). Psychosocial adjustment during pregnancy for older couples conceiving through assisted reproductive technology. Human Reproduction, 22, 1168-1174.

McMahon, C. A., Gibson, F., Leslie, G., Cohen, J., \& Tennant, C. (2003). Parents of 5-year-old in vitro fertilization children: Psychological adjustment, parenting stress and the influence of subsequent in vitro fertilization treatment. Journal of Family Psychology, 17, 361-369.

*McMahon, C., Ungerer, J. A., Beaurepaire, J., Tennant, C., \& Saunders, D. (1997). Anxiety during pregnancy and fetal attachment after in-vitro fertilization conception. Human Reproduction, 12, 176-182.

*Monti, F., Agostini, F., Fagandini, P., La Sala, \& Blickstein, I. (2009). Depressive symptoms during late pregnancy and early parenthood following assisted reproductive technology. Fertility and Sterility, 91, 851-857.

*Monti, F., Agostini, F., Fagandini, P., Paterlini, M., La Sala, G. B., \& Blickstein, I. (2008). Anxiety symptoms during late pregnancy and early parenthood following assisted reproductive technology. Journal of Perinatal Medicine, 36, 425-432.

Passos, E. P., Almeida, I. C. A., \& Fagundes, P. A. P. (2007). Quando a gravidez não acontece. Porto Alegre: Artmed.

*Poikkeus, P., Saisto, T., Unkila-Kallio, L., Punamaki, R. L., Repokari, L., Vilska, S., Tiitnen, A., \& Tulppala, M. (2006). Fear of childbirth and pregnancy-related anxiety in women conceiving with assisted reproduction. Obstetrics \& Gynecology, 108, 70-76.

Raphael-Leff, J. (1997). Gravidez: a história interior. Porto Alegre: Artes Médicas.
*Repokari, L., Punamäki, R. L., Poikkeus, P., Vilska, S., UnkilaKalio, L., Sinkkonen, J., Almqvist, F., Tiitnen, A., \& Tulppala, M. (2005). The impact of successful assisted reproduction treatment on female and male mental health during transition to parenthood: A prospective controlled study. Human Reproduction, 20, 3238-3247.

Ribeiro, M. (2004). Infertilidade e reprodução assistida: Desejando filhos na família contemporânea. São Paulo: Casa do Psicólogo.

Seger-Jacob, L. (2006). Estresse na gênese e no tratamento da infertilidade. Em R. M. M. Melamed \& J. Quayle (Orgs.), Psicologia em reprodução assistida: Experiências brasileiras (pp. 121-153). São Paulo: Casa do Psicólogo.

*Slade, P., Emery, J., \& Lieberman, B. A. (1997). A prospective longitudinal study of emotions and relationship in in-vitro fertilization treatment. Human Reproduction, 12, 183-190.

*Stanton, F., \& Golombok, S. (1993). Maternal-fetal attachment during pregnancy following in vitro fertilization. Journal of Psychosomatic Obstetrics Gynaecology, 14, 153-158.

*Sydsjö, G, Wadsby, M., Kjellberg, S., \& Sydsjo, A. (2002). Relationships and parenthood in couples after assisted reproduction and in spontaneous primiparous couples: A prospective long-term follow-up study. Human Reproduction, 17, 33423250 .

*Ulrich, D., Gagel, D. E., Hemmerling, A., Pastor, V. S., \& Kentenich, H. (2004). Couples becoming parents: Something special after IVF? Journal of Psychosomatic Obstetrics \& Gynecology, 25, 99-113.

*Verhaak, C. M., Smeenk, J. M. J., Eugster, A., Minnen, A., Kremer, J. A. M., \& Kraaimat, F. W. (2001). Stress and marital satisfaction among women before and after their first intracytoplasmatic sprm injection. Fertility and Sterility, 76, 525531.

Verhaak, C. M., Smeenk, J. M. J., Evers, A. W. M., Kremer, J. A. M., Kraaimaat, F. W., \& Braat, D. D. M. (2007). Women's emotional adjustment to IVF: A systematic review of 25 years of research. Human Reproduction Update, 13, 27-36.

*Verhaak, C. M., Smeenk, J. M. J., Minnen, A., Kremer, J. A. M., \& Kraaimat, F. W. (2005). A longitudinal, prospective study on emotional adjustment, before, during and after consecutive fertility treatment cycles. Human Reproduction, 20, 2253-2260.

*Vilska, S., Unkila-Kallio, L., Punämaki, R., L., Poikkeus, P., Repokari, 1., Sinkkonen, J., Tiitnen, A., \& Tulppala, M. (2009). Mental health of mothers and fathers of twins conceived via assisted reproduction treatment: A 1-year prospective study. $\mathrm{Hu}$ man Reproduction, 24, 367-377.

Weiss, T. K. (2006). O impacto da infertilidade e seu tratamento nos casais. Em R. M. M. Melamed \& J. Quayle (Orgs.), Psicologia em reprodução assistida: Experiências brasileiras (pp. 105-119). São Paulo: Casa do Psicólogo.

Recebido: 04/03/2010

Última revisão: $28 / 10 / 2010$ Aceite final: 15/12/2010

\section{Nota:}

${ }^{1}$ Nas demais seções, este agrupamento não foi realizado com o intuito de preservar o foco pretendido pelos autores em cada artigo. 